\title{
Rescattering Effects in the Hadronic-Light-by-Light Contribution to the Anomalous Magnetic Moment of the Muon
}

\author{
Gilberto Colangelo, ${ }^{1}$ Martin Hoferichter, ${ }^{2,3}$ Massimiliano Procura, ${ }^{4, *}$ and Peter Stoffer ${ }^{5,6}$ \\ ${ }^{1}$ Albert Einstein Center for Fundamental Physics, Institute for Theoretical Physics, University of Bern, \\ Sidlerstrasse 5, 3012 Bern, Switzerland \\ ${ }^{2}$ Institute for Nuclear Theory, University of Washington, Seattle, Washington 98195-1550, USA \\ ${ }^{3}$ Kavli Institute for Theoretical Physics, University of California, Santa Barbara, California 93106, USA \\ ${ }^{4}$ Theoretical Physics Department, CERN, 1211 Geneva, Switzerland \\ ${ }^{5}$ Helmholtz-Institut für Strahlen- und Kernphysik (Theory) and Bethe Center for Theoretical Physics, University of Bonn, \\ 53115 Bonn, Germany \\ ${ }^{6}$ Department of Physics, University of California at San Diego, La Jolla, California 92093, USA \\ (Received 26 January 2017; revised manuscript received 14 March 2017; published 9 June 2017)
}

\begin{abstract}
We present a first model-independent calculation of $\pi \pi$ intermediate states in the hadronic-light-by-light (HLBL) contribution to the anomalous magnetic moment of the muon $(g-2)_{\mu}$ that goes beyond the scalar QED pion loop. To this end, we combine a recently developed dispersive description of the HLBL tensor with a partial-wave expansion and demonstrate that the known scalar-QED result is recovered after partialwave resummation. Using dispersive fits to high-statistics data for the pion vector form factor, we provide an evaluation of the full pion box $a_{\mu}^{\pi \text { box }}=-15.9(2) \times 10^{-11}$. We then construct a suitable input for the $\gamma^{*} \gamma^{*} \rightarrow \pi \pi$ helicity partial waves, based on a pion-pole left-hand cut and show that for the dominant charged-pion contribution, this representation is consistent with the two-loop chiral prediction and the COMPASS measurement for the pion polarizability. This allows us to reliably estimate $S$-wave rescattering effects to the full pion box and leads to our final estimate for the sum of these two contributions $a_{\mu}^{\pi \text { box }}+a_{\mu, J=0}^{\pi \pi, \pi \text {-pole LHC }}=-24(1) \times 10^{-11}$.

DOI: 10.1103/PhysRevLett.118.232001
\end{abstract}

Introduction.-The final report of the BNL E821 experiment [1] dominates the world average for the experimental value of the anomalous magnetic moment of the muon $(g-2)_{\mu}$, establishing a departure from its standard-model (SM) expectation by about $3 \sigma$ and thus providing an intriguing hint for new physics that makes the improved measurement at FNAL E989 [2], as well as a potential independent determination at J-PARC E34 [3], highly anticipated (see [4] for a detailed comparison of the two methods). However, the significance of the deviation crucially depends on the details of the SM evaluation. Even more so, a sound interpretation of the future experiments demands that also the theory uncertainties be carefully reassessed and ideally reduced in parallel with the experimental improvement.

The by-far dominant uncertainties in the SM prediction arise from hadronic contributions: hadronic vacuum polarization (HVP) at second order in the fine structure constant $\alpha$ and hadronic-light-by-light scattering (HLBL) at $\mathcal{O}\left(\alpha^{3}\right)$ [5]. With higher-order iterations of the same topologies

Published by the American Physical Society under the terms of the Creative Commons Attribution 4.0 International license. Further distribution of this work must maintain attribution to the author(s) and the published article's title, journal citation, and DOI. already under good control [6-9], most theoretical efforts are concentrated on reducing the uncertainties in the calculations of the HVP and HLBL contributions. But while analyticity and unitarity allow one to express the former in terms of $\sigma\left(e^{+} e^{-} \rightarrow\right.$ hadrons $)[10,11]$, which is well measured, an expression of the HLBL contribution in terms of measurable quantities was not known until recently. So, traditionally, HLBL scattering has been estimated using hadronic models relying on different limits of QCD-large $N_{c}$, chiral symmetry, perturbative expansion-as guiding principles [12-26], which, however, complicates the assessment of the theoretical uncertainty as well as the identification of strategies for systematic improvements, making it emerge as a potential roadblock [27,28].

In a series of recent papers [29-33], we have shown that also the HLBL contribution can be expressed in terms of measurable quantities, albeit not in a form as compact as for HVP. In our model-independent approach based on dispersion relations, we have organized the calculation of the HLBL tensor in terms of its singularities, i.e., singleparticle poles and unitarity cuts, by expanding in the mass of intermediate states [34]. Individual terms in this expansion can be uniquely defined in terms of form factors and scattering amplitudes, which, at least in principle, are accessible to experiment. In this way, the notion of pionpole and pion-box contributions becomes unambiguous, 
and the first terms in the expansion-pseudoscalar poles from $\pi^{0}, \eta, \eta^{\prime}$ intermediate states-are fully determined by the corresponding doubly virtual transition form factors. Progress on the pseudoscalar-pole contributions hinges on improved input for these form factors, in combination with constraints on the asymptotic behavior [23], and only concerns a few of the scalar functions that are necessary for a full description of the HLBL tensor. A program to reconstruct the transition form factors based on a combination of unitarity, analyticity, and perturbative QCD with experimental data is currently under way [41-49].

Next in the expansion are two-pion intermediate states. As demonstrated in [33], the one-loop diagrams evaluated in scalar QED (sQED), including pion vector form factors at each vertex to account for the photon virtuality, provide an exact representation of the contribution of two-pion intermediate states, where only the pion-pole contribution to the left-hand cut (LHC) of the $\gamma^{*} \gamma^{*} \rightarrow \pi \pi$ amplitudes is retained. Thus, the dispersive approach unambiguously defines the gauge-invariant pion-box topology in terms of the pion vector form factor, a very well-measured quantity. Here, we present a numerical evaluation of the pion box, using a form factor fit to high-statistics data, in turn using a dispersive representation to analytically continue the timelike data into the spacelike region required for the $(g-2)_{\mu}$ integral and show that this contribution can be calculated with negligible uncertainties.

Extending our formalism beyond the pion box to account for two-pion rescattering effects is not easy. Here, we briefly review the technical challenges, along with their solutions, to be faced when doing this extension and present a first numerical evaluation of $S$-wave $\pi \pi$-rescattering effects, which unitarize the pion-pole contribution to $\gamma^{*} \gamma^{*} \rightarrow \pi \pi$. This constitutes the first step towards a full treatment of the $\gamma^{*} \gamma^{*} \rightarrow \pi \pi$ partial waves [50-52]. Our calculation settles the role of the pion polarizability, which enters at next-toleading order in the chiral expansion of the HLBL amplitude [53-55] and has been suspected to produce sizable corrections in [54]. In this Letter, we illustrate the general strategy and present first numerical results. Details of the formalism are relegated to [56].

Dispersion relation for HLBL.-The central object in the calculation of the HLBL contribution to $(g-2)_{\mu}$ is the hadronic four-point function

$$
\begin{aligned}
\Pi^{\mu \nu \lambda \sigma}\left(q_{1}, q_{2}, q_{3}\right)= & -i \int d^{4} x d^{4} y d^{4} z e^{-i\left(q_{1} \cdot x+q_{2} \cdot y+q_{3} \cdot z\right)} \\
& \times\left\langle 0\left|T\left\{j_{\mathrm{em}}^{\mu}(x) j_{\mathrm{em}}^{\nu}(y) j_{\mathrm{em}}^{\lambda}(z) j_{\mathrm{em}}^{\sigma}(0)\right\}\right| 0\right\rangle
\end{aligned}
$$

of four electromagnetic currents

$$
j_{\mathrm{em}}^{\mu}=\bar{q} Q \gamma^{\mu} q, \quad Q=\operatorname{diag}\left(\frac{2}{3},-\frac{1}{3},-\frac{1}{3}\right),
$$

with momenta $q_{i}$ as indicated, $q_{4}=q_{1}+q_{2}+q_{3}$, and quark fields $q=(u, d, s)^{T}$.

To be able to reconstruct the HLBL tensor $\Pi^{\mu \nu \lambda \sigma}$ with dispersion relations, it is imperative to use a decomposition into scalar functions that are free of kinematic singularities and zeros. Such a representation can be obtained following the general recipe put forward by Bardeen, Tung [57], and Tarrach [58] (BTT), resulting in

$$
\Pi^{\mu \nu \lambda \sigma}=\sum_{i=1}^{54} T_{i}^{\mu \nu \lambda \sigma} \Pi_{i},
$$

with scalar functions $\Pi_{i}$ depending on the Mandelstam variables $s=\left(q_{1}+q_{2}\right)^{2}, t=\left(q_{1}+q_{3}\right)^{2}, u=\left(q_{2}+q_{3}\right)^{2}$ as well as the virtualities $q_{i}^{2}$ and Lorentz structures $T_{i}^{\mu \nu \lambda \sigma}$ $[32,33]$. This decomposition fulfills gauge invariance manifestly

$$
\left\{q_{1}^{\mu}, q_{2}^{\nu}, q_{3}^{\lambda}, q_{4}^{\sigma}\right\} T_{\mu \nu \lambda \sigma}^{i}=0,
$$

is highly crossing symmetric (with only 7 distinct structures, all remaining 47 being related to these by crossing transformations), and ensures that the coefficient functions $\Pi_{i}$ do not contain kinematic singularities and zeros. In addition, the BTT decomposition typically allows for a very economical representation of HLBL amplitudes; e.g., one of the structures coincides with the amplitude for a pseudoscalar pole, while even the SQED amplitude becomes very compact once expressed in terms of BTT functions [56]. For the contribution to $(g-2)_{\mu}$, a threedimensional integral representation is available [56]

$$
\begin{aligned}
a_{\mu}^{\mathrm{HLBL}}= & \frac{\alpha^{3}}{432 \pi^{2}} \int_{0}^{\infty} d \Sigma \Sigma^{3} \int_{0}^{1} d r r \sqrt{1-r^{2}} \int_{0}^{2 \pi} d \phi \\
& \times \sum_{i=1}^{12} T_{i}\left(Q_{1}, Q_{2}, Q_{3}\right) \bar{\Pi}_{i}\left(Q_{1}, Q_{2}, Q_{3}\right),
\end{aligned}
$$

where the $T_{i}$ are known kernel functions, the $\bar{\Pi}_{i}$ suitable linear combinations of the BTT $\Pi_{i}$, and the Euclidean momenta squared are given by [59]

$$
\begin{aligned}
Q_{1,2}^{2} & =\frac{\Sigma}{3}\left(1-\frac{r}{2} \cos \phi \mp \frac{r}{2} \sqrt{3} \sin \phi\right), \\
Q_{3}^{2} & =\frac{\Sigma}{3}(1+r \cos \phi) .
\end{aligned}
$$

There are only 6 distinct functions $\bar{\Pi}_{i}$; the remaining ones are again related to these by crossing symmetry. It suffices to calculate the $\bar{\Pi}_{i}$ in the kinematic limit, where $q_{4} \rightarrow 0$; the transition to $(g-2)_{\mu}$ then proceeds by means of (5).

Two-pion intermediate states. - In a dispersive approach, two-pion intermediate states comprise all contributions that involve a two-pion cut, generically represented by the left (unitarity) diagram in Fig. 1. The dominant term is obtained if in the $\gamma^{*} \gamma^{*} \rightarrow \pi \pi$ subamplitudes, in turn, the pion is put on shell, i.e., if the pion-pole contribution to the LHC is 
(a)

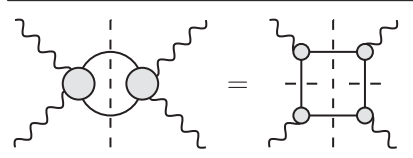

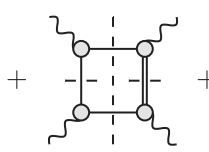

(b)

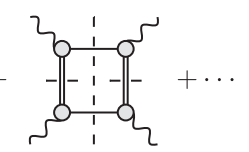

(c)
FIG. 1. Two-pion-cut contributions to HLBL scattering. Solid (wiggly) lines refer to pions (photons), while dashed lines indicate the cutting of propagators. Double lines generically denote heavier intermediate states, the gray blobs hadronic amplitudes. Crossed diagrams are omitted.

isolated. In this case, the remaining hadronic amplitudes are given by pion vector form factors, and as demonstrated in [33], this class of two-pion intermediate states, the pure pion box in diagram $(a)$ in Fig. 1, reproduces the sQED pion loop with vertices augmented by the appropriate pion form factors. The reason for this behavior can be traced back to the fact that only the singularities of the box diagrams in SQED matter, while the triangle and bulb diagrams are simply required to restore gauge invariance. Because of the high degree of crossing symmetry, this pionbox contribution can be expressed in terms of either fixed$s$, $-t$, or $-u$ dispersion relations or in a symmetrized form

$$
\begin{aligned}
\Pi_{i}^{\pi \operatorname{box}}(s, t, u)= & \frac{1}{3}\left(\frac{1}{\pi} \int_{4 M_{\pi}^{2}}^{\infty} d t^{\prime} \frac{\operatorname{Im} \Pi_{i}^{\pi \operatorname{box}}\left(s, t^{\prime}, u^{\prime}\right)}{t^{\prime}-t}\right. \\
& +\frac{1}{\pi} \int_{4 M_{\pi}^{2}}^{\infty} d u^{\prime} \frac{\operatorname{Im} \Pi_{i}^{\pi \operatorname{box}}\left(s, t^{\prime}, u^{\prime}\right)}{u^{\prime}-u} \\
& + \text { fixed } t+\text { fixed } u) .
\end{aligned}
$$

In this case, the representation is exact.

Once heavier intermediate states are considered, generically denoted by the double lines in diagrams $(b)$ and $(c)$ in Fig. 1, a more detailed investigation of the double spectral functions is required. In practice, such contributions can be included using a partial-wave expansion, in which case the subprocess becomes a polynomial in the crossed variable, and the crossed-channel cuts are neglected. Writing down all crossed versions of the unitarity diagrams shown in Fig. 1, one sees that each double spectral region appears exactly twice in a symmetrized form as in (7) so that the prefactor has to be changed from $1 / 3 \rightarrow 1 / 2$ [56], with corrections suppressed by the mass scale of the neglected LHC. In particular, this representation becomes exact for $\pi \pi$-rescattering effects, which, by definition, are polynomial in the crossed Mandelstam variable.

Partial-wave expansion.-Constraints from unitarity are most conveniently formulated in a partial-wave expansion for HLBL helicity amplitudes $h_{\lambda_{1} \lambda_{2}, \lambda_{3} \lambda_{4}}^{J}$, with angular momentum $J$ and helicity labels $\lambda_{i}$. In this case, the unitarity relation becomes diagonal

$$
\operatorname{Im} h_{\lambda_{1} \lambda_{2}, \lambda_{3} \lambda_{4}}^{J}(s)=\frac{\sigma_{\pi}(s)}{16 \pi S} h_{J, \lambda_{1} \lambda_{2}}(s) h_{J, \lambda_{3} \lambda_{4}}^{*}(s),
$$

where $\sigma_{\pi}(s)=\sqrt{1-4 M_{\pi}^{2} / s}$ gives the phase space, $S=2$ a symmetry factor in case of indistinguishable particles, and $h_{J, \lambda_{1} \lambda_{2}}$ the helicity partial waves for $\gamma^{*} \gamma^{*} \rightarrow \pi \pi$. Once formulated in isospin basis, Watson's theorem [60] [see also (10) below] guarantees that the phases on the righthand side cancel to produce a real imaginary part. The partial-wave expansion of the pion box is obtained if both $h_{J, \lambda_{1} \lambda_{2}}(s)$ and $h_{J, \lambda_{3} \lambda_{4}}(s)$ are identified with the partial-waveprojected Born terms, while the rescattering effects correspond to the unitarity corrections to either subamplitude derived from (10).

There are 41 independent helicity amplitudes for the full HLBL tensor, which reduce to 27 if one photon is taken on shell. Rewriting the representation of the contribution to $(g-2)_{\mu}$, Eq. (5), in such a way that only dispersive integrals over imaginary parts of these 27 helicity amplitudes appear is highly nontrivial. By explicitly requiring that unphysical amplitudes drop out in the final result and that the two redundancies which appear in four space-time dimensions $d=4$ [59] do not affect the result, one can derive a set of sum rules for the scalar functions. (In [61], sum rules for the special case of forward HLBL scattering have been derived.) These sum rules apply to the full amplitudes but not necessarily at the level of the partialwave-expanded ones, producing an apparent dependence on unphysical amplitudes that would only disappear after a resummation of all partial waves.

To avoid such pathologies, we were able to construct a set of 27 amplitudes $\check{\Pi}_{i}$, related to the 27 singly on-shell helicity amplitudes by a basis change that we have derived in explicit analytic form. In the limit $q_{4} \rightarrow 0$, a subset of the $\check{\Pi}_{i}$ includes all the scalar functions needed as input in (5) [56]. Moreover, this set of 27 amplitudes is manifestly free of Tarrach [58] or $d=4$ ambiguities [59]. For singly onshell kinematics, there still exist 15 sum rules among the 27 helicity amplitudes, which we have exploited to optimize to a certain degree the representation with respect to the convergence of the partial-wave expansion. This formalism is now ready to be applied to the evaluation of rescattering effects, but before doing that, we test it with the help of the pion box and study how well we are able to reproduce its numerical value by resumming the partial-wave expansion.

Pion box.-The formalism for dealing with the pion box has been developed in [33]. Here, we provide a first numerical evaluation thereof, with a realistic pion form factor. The latter has been obtained by fitting a dispersive representation as suggested in $[62,63]$ to both spacelike [64] and timelike [65-70] form factor data (similar representations have been used before in [71-76]), with the result

$$
a_{\mu}^{\pi \mathrm{box}}=-15.9(2) \times 10^{-11},
$$

and an uncertainty determined from the differences between the timelike data sets as well as the details of the fit representation. The main reduction in uncertainty compared 
to earlier evaluations of a "pion loop" $[13,16]$ is due to the insight that the pion box, defined as two-pion intermediate states with a pion-pole left-hand cut, is the unambiguous first term in the expansion and can be expressed in terms of a hadronic observable, the pion vector form factor, which is very well known phenomenologically.

The pion box also provides an ideal test case for the framework presented in the previous section since the full result is known, and explicit expressions for all BTT scalar functions are available. As a first step, we verified that the sum rules encountered in the context of the partial-wave expansion are fulfilled. Second, in the special case of the pion box, a fixed- $s,-t,-u$ representation should each hold, combining to the symmetrized version in (7), so that the convergence can be studied in each channel separately. The results, for simplicity, obtained by using a vector-mesondominance pion form factor $F_{\pi}^{V}\left(q^{2}\right)=M_{\rho}^{2} /\left(M_{\rho}^{2}-q^{2}\right)$, with $a_{\mu}^{\pi \text { box,VMD }}=-16.4 \times 10^{-11}$, are shown in Table I, demonstrating that each representation approaches the full result (going up to $J_{\max }=20$, we checked that also the remaining differences disappear after partial-wave resummation). The vanishing $S$-wave contribution for fixed $s$ is well understood and partly a matter of convention in the choice of the six functions $\bar{\Pi}_{i}$, see [56]. In concrete applications, the prescription of changing the prefactor in (7), as explained above, combines the three representations in a way that best captures the physics (such as a resonance) in all channels at once, which means that the convergence patterns for fixed $t$ or $-u$ are more representative of realistic cases, and the average of the three should be viewed as a worst-case scenario. But even that displays a very reasonable convergence behavior.

$\pi \pi$-rescattering effects. -We now turn to the evaluation of rescattering effects as a first important step to go beyond the pion-box contribution. The helicity amplitudes $h_{J, \lambda_{1} \lambda_{2}}(s)$ entering (8) satisfy themselves a unitarity relation

$$
\operatorname{Im} h_{J, \lambda_{1} \lambda_{2}}^{I}(s)=\sin \delta_{J}^{I}(s) e^{-i \delta_{J}^{I}(s)} h_{J, \lambda_{1} \lambda_{2}}^{I}(s),
$$

with isospin labels $I$ and $\pi \pi$ phase shifts $\delta_{J}^{I}$. This relation is clearly violated for the (real) Born terms alone, but this deficiency can be easily repaired by solving the dispersion relation for the subprocess $\gamma^{*} \gamma^{*} \rightarrow \pi \pi$.

TABLE I. Saturation of $a_{\mu}^{\pi \text { box }}$ for maximal angular momentum $J_{\text {max }}$.

\begin{tabular}{lccrc}
\hline \hline$J_{\max }$ & Fixed $s$ & Fixed $t$ & Fixed $u$ & Average \\
\hline 0 & $0.0 \%$ & $106.2 \%$ & $106.2 \%$ & $70.8 \%$ \\
2 & $73.9 \%$ & $102.3 \%$ & $92.7 \%$ & $89.6 \%$ \\
4 & $89.2 \%$ & $101.5 \%$ & $96.4 \%$ & $95.7 \%$ \\
6 & $94.3 \%$ & $100.7 \%$ & $97.9 \%$ & $97.6 \%$ \\
8 & $96.5 \%$ & $100.4 \%$ & $98.7 \%$ & $98.5 \%$ \\
\hline \hline
\end{tabular}

In contrast to the on-shell and singly virtual case [5052], the calculation of the $\gamma^{*} \gamma^{*} \rightarrow \pi \pi$ partial waves for two off-shell photons is complicated by the fact that even for $S$ waves two different helicity partial waves, $h_{0,++}$ and $h_{0,00}$, become coupled, including off-diagonal kernel functions required to eliminate kinematic singularities [30,33]. Here, we apply this framework to construct the $\gamma^{*} \gamma^{*} \rightarrow \pi \pi$ amplitudes that correspond to the rescattering corrections to the Born terms, whose solution can still be derived based on Muskhelishvili-Omnès methods [77,78]. We use $\pi \pi$ phase shifts, based on the modified inverse-amplitude method [79], for the main reason that it has a simple analytic expression, which is convenient to use in combination with Muskhelishvili-Omnès methods, while at the same time, it reproduces accurately the low-energy properties of the phase shifts as well as pole position and couplings of the $f_{0}(500)$ resonance. This phase shift departs from the correct one just below the $K \bar{K}$ threshold because it does not feature the sharp rise due to the $f_{0}(980)$ resonance but continues flat with a smooth high-energy behavior. A full-fledged evaluation of the $f_{0}(980)$ resonance would require a proper treatment of the $K \bar{K}$ channel, which is beyond the scope of this first estimate. We can, on the other hand, test the sensitivity to the asymptotic part of the dispersive integrals by studying solutions with different cutoff values $\Lambda=[1 \mathrm{GeV}, \infty)$, constructed with finitematching-point techniques [51,80-83]. Moreover, we checked that for low values of $\Lambda$, phase shifts obtained by solving Roy equations [84-86] lead to equivalent results.

The results for the rescattering contribution, summarized in Table II, are indeed stable over a wide range of cutoffs, indicating that our input for the $\gamma^{*} \gamma^{*} \rightarrow \pi \pi$ partial waves reliably unitarizes the Born-term LHC, which should indeed dominate at low energies. In addition, we checked that the only sum rule that receives $S$-wave contributions is already saturated at better than $90 \%$, completely in line with the expectation that the sum rules will be fulfilled only after partial-wave resummation. The isospin- 0 part of the result can be interpreted as a model-independent implementation of the contribution from the $f_{0}(500)$ of about $-9 \times 10^{-11}$ to HLBL scattering in $(g-2)_{\mu}$. In total, we obtain for the $\pi \pi$-rescattering effects related to the pion-pole LHC,

$$
a_{\mu, J=0}^{\pi \pi, \pi \text {-pole LHC }}=-8(1) \times 10^{-11}
$$

TABLE II. $S$-wave rescattering corrections to $a_{\mu}^{\pi \text { box }}$ in units of $10^{-11}$ for both isospin components and in total.

\begin{tabular}{lrcrr}
\hline \hline Cutoff & $1 \mathrm{GeV}$ & $1.5 \mathrm{GeV}$ & $2 \mathrm{GeV}$ & \multicolumn{1}{c}{$\infty$} \\
\hline$I=0$ & -9.2 & -9.5 & -9.3 & -8.8 \\
$I=2$ & 2.0 & 1.3 & 1.1 & 0.9 \\
Sum & -7.3 & -8.3 & -8.3 & -7.9 \\
\hline \hline
\end{tabular}


where the error is dominated by the uncertainties related to the asymptotic parts of the integral, see Table II. Improving the energy region $\gtrsim 1 \mathrm{GeV}$ requires the inclusion of the $K \bar{K}$ channel as well as higher contributions to the LHC, neither of which can be expressed in terms of the pion vector form factor. Very likely, such effects beyond pion states will be less precisely estimated.

Finally, it is instructive to consider the separate contributions not in the isospin but in the charge basis. In this case, the unitarity relation (8) is no longer diagonal, and it is not possible to define unambiguously the contribution of each of the charge states. Irrespective of the detailed convention for the separation, charged-pion states are expected to strongly dominate; e.g., in the chiral expansion, neutral-pion intermediate states first appear at three-loop order. The derivative of the Born-term-subtracted amplitude $h_{0,++}(s)$ is related to the pion dipole polarizability $\alpha_{1}-\beta_{1}$, to which the unitarized pion-pole LHC contributes

$$
\begin{aligned}
\left(\alpha_{1}-\beta_{1}\right)^{\pi^{ \pm}, \pi \text {-pole LHC }}=(5.4 \ldots 5.8) \times 10^{-4} \mathrm{fm}^{3}, \\
\left(\alpha_{1}-\beta_{1}\right)^{\pi^{0}, \pi \text {-pole LHC }}=(11.2 \ldots 8.9) \times 10^{-4} \mathrm{fm}^{3}
\end{aligned}
$$

for $\Lambda=1 \mathrm{GeV} \ldots \infty$. For the charged pion, this result is in perfect agreement with the chiral two-loop prediction 5.7(1.0) [87] (in the same units) as well as the recent COMPASS measurement 4.0(1.2) $)_{\text {stat }}(1.4)_{\text {syst }}$ [88]. In contrast, the two-loop prediction for the neutral pion $-1.9(0.2)$ [89] is substantially smaller in size and has the opposite sign of what we get from our representation. This failure, however, is not reason for much concern because we are not yet including here the main contributions to the LHC of the amplitude for neutral pions, i.e., vector-meson exchange involving $V=\omega, \rho$. Because of the scaling with $\Gamma_{V \rightarrow \pi \gamma}$, the relative impact on the neutral channel [90]

$$
\frac{\Gamma_{\omega} \times \mathrm{BR}\left[\omega \rightarrow \pi^{0} \gamma\right]+\Gamma_{\rho} \times \mathrm{BR}\left[\rho^{0} \rightarrow \pi^{0} \gamma\right]}{\Gamma_{\rho} \times \mathrm{BR}\left[\rho^{ \pm} \rightarrow \pi^{ \pm} \gamma\right]} \sim 12
$$

is an order of magnitude larger so that heavier intermediate states allow one to repair $\left(\alpha_{1}-\beta_{1}\right)^{\pi^{0}}$ without spoiling agreement in the charged channel. In summary, the rescattering effects in (11) are dominated by the charged pion, with input for the $\gamma^{*} \gamma^{*} \rightarrow \pi \pi$ partial waves fully consistent with its dipole polarizability. For this reason, (11) can be considered a model-independent implementation of effects related to the low-energy constants $L_{9}$ and $L_{10}$, which were suspected to produce large effects in [54]. Our calculation proves that this is not the case and that the related rescattering corrections are indeed of very reasonable size (a similar conclusion was reached within a model approach in [55]). In this context, it should be stressed that our analysis does not rely on chiral operators, thus avoiding the pathologies in their high-energy behavior and the need to cure them. The polarizabilities enter here as the limit of our $\gamma^{*} \gamma^{*} \rightarrow \pi \pi$ amplitudes at a particular kinematic point that does not contribute to the dispersive integrals directly, providing an important cross-check of the low-energy limit.

In conclusion, we have shown that our framework allows us to estimate, very accurately, the combined effect of twopion intermediate states generated by a pion-pole LHC and its $S$-wave unitarization

$$
a_{\mu}^{\pi \text { box }}+a_{\mu, J=0}^{\pi \pi, \pi \text {-pole LHC }}=-24(1) \times 10^{-11},
$$

which is considered to be among the most important contributions after the dominant pseudoscalar poles but was so far affected by significant uncertainties. This first numerical result based on the dispersive approach lays the foundation for extensions towards higher partial waves, an improved LHC in the $\gamma^{*} \gamma^{*} \rightarrow \pi \pi$ subamplitudes, as well as higher-mass intermediate states, all important prerequisites for a model-independent evaluation of the complete HLBL contribution to $(g-2)_{\mu}$.

Financial support by the DFG (SFB/TR 16, "Subnuclear Structure of Matter," SFB/TR 110, "Symmetries and the Emergence of Structure in QCD"), the DOE (Grant No. DE-FG02-00ER41132), the National Science Foundation (Grant No. NSF PHY-1125915), and the Swiss National Science Foundation is gratefully acknowledged. M. P. is supported by a Marie Curie Intra-European Fellowship of the European Community's 7th Framework Programme under Contract No. PIEF-GA-2013-622527 and P.S. by a grant of the Swiss National Science Foundation (Project No. P300P2_167751).

* On leave from the University of Vienna.

[1] G. W. Bennett et al. (Muon g-2 Collaboration), Phys. Rev. D 73, 072003 (2006).

[2] J. Grange et al. (Muon g-2 Collaboration), arXiv: 1501.06858.

[3] N. Saito (J-PARC g-2/EDM Collaboration), AIP Conf. Proc. 1467, 45 (2012).

[4] T. P. Gorringe and D. W. Hertzog, Prog. Part. Nucl. Phys. 84, 73 (2015).

[5] F. Jegerlehner and A. Nyffeler, Phys. Rep. 477, 1 (2009).

[6] J. Calmet, S. Narison, M. Perrottet, and E. de Rafael, Phys. Lett. 61B, 283 (1976).

[7] K. Hagiwara, R. Liao, A. D. Martin, D. Nomura, and T. Teubner, J. Phys. G 38, 085003 (2011).

[8] A. Kurz, T. Liu, P. Marquard, and M. Steinhauser, Phys. Lett. B 734, 144 (2014).

[9] G. Colangelo, M. Hoferichter, A. Nyffeler, M. Passera, and P. Stoffer, Phys. Lett. B 735, 90 (2014).

[10] C. Bouchiat and L. Michel, J. Phys. Radium 22, 121 (1961).

[11] T. Blum, A. Denig, I. Logashenko, E. de Rafael, B. Lee Roberts, T. Teubner, and G. Venanzoni, arXiv:1311.2198.

[12] E. de Rafael, Phys. Lett. B 322, 239 (1994).

[13] J. Bijnens, E. Pallante, and J. Prades, Phys. Rev. Lett. 75, 1447 (1995); 75, 3781(E) (1995). 
[14] J. Bijnens, E. Pallante, and J. Prades, Nucl. Phys. B474, 379 (1996).

[15] J. Bijnens, E. Pallante, and J. Prades, Nucl. Phys. B626, 410 (2002).

[16] M. Hayakawa, T. Kinoshita, and A. I. Sanda, Phys. Rev. Lett. 75, 790 (1995).

[17] M. Hayakawa, T. Kinoshita, and A. I. Sanda, Phys. Rev. D 54, 3137 (1996).

[18] M. Hayakawa and T. Kinoshita, Phys. Rev. D 57, 465 (1998); 66, 019902(E) (2002).

[19] A. A. Pivovarov, Yad. Fiz. 66, 934 (2003) [Phys. At. Nucl. 66, 902 (2003)].

[20] M. Knecht, A. Nyffeler, M. Perrottet, and E. de Rafael, Phys. Rev. Lett. 88, 071802 (2002).

[21] M. Knecht and A. Nyffeler, Phys. Rev. D 65, 073034 (2002).

[22] M. J. Ramsey-Musolf and M. B. Wise, Phys. Rev. Lett. 89, 041601 (2002).

[23] K. Melnikov and A. Vainshtein, Phys. Rev. D 70, 113006 (2004).

[24] J. Erler and G. Toledo Sánchez, Phys. Rev. Lett. 97, 161801 (2006).

[25] T. Goecke, C. S. Fischer, and R. Williams, Phys. Rev. D 83, 094006 (2011); 86, 099901(E) (2012).

[26] D. Greynat and E. de Rafael, J. High Energy Phys. 07 (2012) 020.

[27] J. Prades, E. de Rafael, and A. Vainshtein, Adv. Ser. Dir. High Energy Phys. 20, 303 (2009).

[28] M. Benayoun et al., arXiv:1407.4021.

[29] M. Hoferichter, G. Colangelo, M. Procura, and P. Stoffer, Int. J. Mod. Phys. Conf. Ser. 35, 1460400 (2014).

[30] G. Colangelo, M. Hoferichter, M. Procura, and P. Stoffer, J. High Energy Phys. 09 (2014) 091.

[31] G. Colangelo, M. Hoferichter, B. Kubis, M. Procura, and P. Stoffer, Phys. Lett. B 738, 6 (2014).

[32] P. Stoffer, arXiv:1412.5171.

[33] G. Colangelo, M. Hoferichter, M. Procura, and P. Stoffer, J. High Energy Phys. 09 (2015) 074.

[34] A dispersive approach for the Pauli form factor instead of the HLBL tensor has been suggested in [35]. The lattice QCD approach, complementary to improved phenomenological calculations, is also promising, see [36-40].

[35] V. Pauk and M. Vanderhaeghen, Phys. Rev. D 90, 113012 (2014).

[36] T. Blum, S. Chowdhury, M. Hayakawa, and T. Izubuchi, Phys. Rev. Lett. 114, 012001 (2015).

[37] J. Green, O. Gryniuk, G. von Hippel, H. B. Meyer, and V. Pascalutsa, Phys. Rev. Lett. 115, 222003 (2015).

[38] T. Blum, N. Christ, M. Hayakawa, T. Izubuchi, L. Jin, and C. Lehner, Phys. Rev. D 93, 014503 (2016).

[39] A. Gérardin, H. B. Meyer, and A. Nyffeler, Phys. Rev. D 94, 074507 (2016).

[40] T. Blum, N. Christ, M. Hayakawa, T. Izubuchi, L. Jin, C. Jung, and C. Lehner, Phys. Rev. Lett. 118, 022005 (2017).

[41] F. Stollenwerk, C. Hanhart, A. Kupść, U.-G. Meißner, and A. Wirzba, Phys. Lett. B 707, 184 (2012).

[42] F. Niecknig, B. Kubis, and S. P. Schneider, Eur. Phys. J. C 72, 2014 (2012).

[43] S. P. Schneider, B. Kubis, and F. Niecknig, Phys. Rev. D 86, 054013 (2012).
[44] M. Hoferichter, B. Kubis, and D. Sakkas, Phys. Rev. D 86, 116009 (2012).

[45] C. Hanhart, A. Kupść, U.-G. Meißner, F. Stollenwerk, and A. Wirzba, Eur. Phys. J. C 73, 2668 (2013); 75, 242(E) (2015).

[46] M. Hoferichter, B. Kubis, S. Leupold, F. Niecknig, and S. P. Schneider, Eur. Phys. J. C 74, 3180 (2014).

[47] B. Kubis and J. Plenter, Eur. Phys. J. C 75, 283 (2015).

[48] C. W. Xiao, T. Dato, C. Hanhart, B. Kubis, U.-G. Meißner, and A. Wirzba, arXiv:1509.02194.

[49] A. Nyffeler, Phys. Rev. D 94, 053006 (2016).

[50] R. García-Martín and B. Moussallam, Eur. Phys. J. C 70, 155 (2010).

[51] M. Hoferichter, D. R. Phillips, and C. Schat, Eur. Phys. J. C 71, 1743 (2011).

[52] B. Moussallam, Eur. Phys. J. C 73, 2539 (2013).

[53] K. T. Engel, H. H. Patel, and M. J. Ramsey-Musolf, Phys. Rev. D 86, 037502 (2012).

[54] K. T. Engel and M. J. Ramsey-Musolf, Phys. Lett. B 738, 123 (2014).

[55] J. Bijnens and J. Relefors, J. High Energy Phys. 09 (2016) 113.

[56] G. Colangelo, M. Hoferichter, M. Procura, and P. Stoffer, J. High Energy Phys. 04 (2017) 161.

[57] W. A. Bardeen and W. K. Tung, Phys. Rev. 173, 1423 (1968); 4, 3229(E) (1971).

[58] R. Tarrach, Nuovo Cimento Soc. Ital. Fis. A28, 409 (1975).

[59] G. Eichmann, C. S. Fischer, and W. Heupel, Phys. Rev. D 92, 056006 (2015).

[60] K. M. Watson, Phys. Rev. 95, 228 (1954).

[61] V. Pascalutsa, V. Pauk, and M. Vanderhaeghen, Phys. Rev. D 85, 116001 (2012).

[62] H. Leutwyler, arXiv:hep-ph/0212324.

[63] G. Colangelo, Nucl. Phys. B, Proc. Suppl. 131, 185 (2004).

[64] S. R. Amendolia et al. (NA7 Collaboration), Nucl. Phys. B277, 168 (1986).

[65] M. N. Achasov et al., Zh. Eksp. Teor. Fiz. 130, 437 (2006) [J. Exp. Theor. Phys. 103, 380 (2006)].

[66] R. R. Akhmetshin et al. (CMD-2 Collaboration), Phys. Lett. B 648, 28 (2007).

[67] B. Aubert et al. (BABAR Collaboration), Phys. Rev. Lett. 103, 231801 (2009).

[68] F. Ambrosino et al. (KLOE Collaboration), Phys. Lett. B 700, 102 (2011).

[69] D. Babusci et al. (KLOE Collaboration), Phys. Lett. B 720, 336 (2013).

[70] M. Ablikim et al. (BESIII Collaboration), Phys. Lett. B 753, 629 (2016).

[71] J. F. de Trocóniz and F. J. Ynduráin, Phys. Rev. D 65, 093001 (2002).

[72] J. F. de Trocóniz and F. J. Ynduráin, Phys. Rev. D 71, 073008 (2005).

[73] B. Ananthanarayan, I. Caprini, D. Das, and I. S. Imsong, Phys. Rev. D 89, 036007 (2014).

[74] B. Ananthanarayan, I. Caprini, D. Das, and I. S. Imsong, Phys. Rev. D 93, 116007 (2016).

[75] M. Hoferichter, B. Kubis, J. Ruiz de Elvira, H.-W. Hammer, and U.-G. Meißner, Eur. Phys. J. A 52, 331 (2016).

[76] C. Hanhart, S. Holz, B. Kubis, A. Kupść, A. Wirzba, and C. W. Xiao, Eur. Phys. J. C 77, 98 (2017). 
[77] N. I. Muskhelishvili, Singular Integral Equations, (WoltersNoordhoff Publishing, Groningen, 1953) (republished by Dover Publications, Mineola, NY, 2008).

[78] R. Omnès, Nuovo Cimento 8, 316 (1958).

[79] A. Gómez Nicola, J. R. Peláez, and G. Ríos, Phys. Rev. D 77, 056006 (2008).

[80] P. Büttiker, S. Descotes-Genon, and B. Moussallam, Eur. Phys. J. C 33, 409 (2004).

[81] C. Ditsche, M. Hoferichter, B. Kubis, and U.-G. Meißner, J. High Energy Phys. 06 (2012) 043.

[82] M. Hoferichter, C. Ditsche, B. Kubis, and U.-G. Meißner, J. High Energy Phys. 06 (2012) 063.

[83] M. Hoferichter, J. Ruiz de Elvira, B. Kubis, and U.-G. Meißner, Phys. Rep. 625, 1 (2016).
[84] G. Colangelo, J. Gasser, and H. Leutwyler, Nucl. Phys. B603, 125 (2001).

[85] I. Caprini, G. Colangelo, and H. Leutwyler, Eur. Phys. J. C 72, 1860 (2012).

[86] R. García-Martín, R. Kamiński, J. R. Peláez, J. Ruiz de Elvira, and F. J. Ynduráin, Phys. Rev. D 83, 074004 (2011).

[87] J. Gasser, M. A. Ivanov, and M. E. Sainio, Nucl. Phys. B745, 84 (2006).

[88] C. Adolph et al. (COMPASS Collaboration), Phys. Rev. Lett. 114, 062002 (2015).

[89] J. Gasser, M. A. Ivanov, and M. E. Sainio, Nucl. Phys. B728, 31 (2005).

[90] C. Patrignani et al. (Particle Data Group Collaboration), Chin. Phys. C 40, 100001 (2016). 